\title{
ISOMORPHISMS PRESERVING INVARIANTS
}

\author{
GERALD W. SCHWARZ
}

\begin{abstract}
Let $V$ and $W$ be finite dimensional real vector spaces and let $G \subset \operatorname{GL}(V)$ and $H \subset \mathrm{GL}(W)$ be finite subgroups. Assume for simplicity that the actions contain no reflections. Let $Y$ and $Z$ denote the real algebraic varieties corresponding to $\mathbb{R}[V]^{G}$ and $\mathbb{R}[W]^{H}$, respectively. If $V$ and $W$ are quasi-isomorphic, i.e., if there is a linear isomorphism $L: V \rightarrow W$ such that $L$ sends $G$-orbits to $H$-orbits and $L^{-1}$ sends $H$-orbits to $G$-orbits, then $L$ induces an isomorphism of $Y$ and $Z$. Conversely, suppose that $f: Y \rightarrow Z$ is a germ of a diffeomorphism sending the origin of $Y$ to the origin of $Z$. Then we show that $V$ and $W$ are quasi-isomorphic, This result is closely related to a theorem of Strub [Str82], for which we give a new proof. We also give a new proof of a result of [KLM03] on lifting of biholomorphisms of quotient spaces.
\end{abstract}

\section{INTRODUCTION}

First some motivation. Let $G$ be a finite group and let $M$ and $N$ be smooth $G$-manifolds. We give the orbit spaces $M / G$ and $N / G$ a smooth structure by declaring that $C^{\infty}(M / G)=C^{\infty}(M)^{G}$ and $C^{\infty}(N / G)=C^{\infty}(N)^{G}$. We also have a stratification of $M / G$ by isotropy type, where the type of a point $G m$ of $M / G$ is the conjugacy class of the isotropy group $G_{m}$ of $m \in M$. Suppose that $M$ and $N$ are equivariantly diffeomorphic. Then $M / G$ and $N / G$ are "diffeomorphic" and the diffeomorphism preserves isotropy type. Conversely, suppose that $M / G$ and $N / G$ are diffeomorphic by a strata preserving diffeomorphism $f$. Can we lift $f$ to an equivariant diffeomorphism of $M$ and $N$ ? First of all, $f$ must give a diffeomorphism of $M^{G}$ and $N^{G}$ (assuming these sets are nonempty). Let $v \in M^{G}$ and set $V=T_{v} M$. Let $f(v)=w \in N^{G}$ and set $W=T_{w} N$. Then the differentiable slice theorem says that there is a neighborhood of $v$ in $M / G$ diffeomorphic to $U \times V / G$ where $U$ is a neighborhood of $v$ in $M^{G}$. It follows that $f$ induces a strata preserving diffeomorphism of $V / G$ with $W / G$. The first question to answer is if the existence of a diffeomorphism of $V / G$ with $W / G$ implies that $V$ and $W$ are isomorphic representations of $G$. The answer is "yes," up to an automorphism of $G$, by a theorem of Strub [Str82]. We generalize the problem by replacing the requirement that strata are preserved by a weaker condition, we allow for a different group $H$ to act on $W$ and we allow the diffeomorphism $f$ to be defined on the Zariski closures of the orbit spaces, in a sense made precise below. This ends our motivation, and the precise description of our results follows.

Let $V$ and $W$ be finite dimensional real vector spaces and let $G \subset \mathrm{GL}(V)$ and $H \subset \mathrm{GL}(W)$ be finite subgroups. Let $Y$ and $Z$ denote the real algebraic varieties corresponding to $\mathbb{R}[V]^{G}$ and $\mathbb{R}[W]^{H}$, respectively. We say that a linear isomorphism $L: V \rightarrow W$ is a quasi-isomorphism if $L$ sends $G$-orbits to $H$-orbits and $L^{-1}$ sends $H$-orbits to $G$-orbits. We say that $V$ and $W$ are quasi-isomorphic if such an $L$ exists. If $L$ is a quasi-isomorphism, then $g \mapsto L \circ g \circ L^{-1}$ gives an isomorphism of $G$ and $H$ such that, with $G$ acting on $W$ via the isomorphism, $L$ is equivariant.

If $V$ and $W$ are quasi-isomorphic, then $Y$ and $Z$ are isomorphic. Conversely, we show that if $f: Y \rightarrow Z$ is a germ of a diffeomorphism sending the origin of $Y$ to the origin of $Z$ such that $f$ maps the closures of the codimension one strata of $Y$ onto those of $Z$, then $V$ and $W$ are quasi-isomorphic. The origin of $Y$ is the image of $0 \in V$ in $Y$ and similarly for $Z$.

2000 Mathematics Subject Classification. 20G20, 14L30.

Key words and phrases. Invariant polynomials.

Partially supported by NSA Grant H98230-06-1-0023. 
There are closely related results in the literature. Let $Y_{0}$ and $Z_{0}$ denote the images of $V$ and $W$ in $Y$ and $Z$, respectively. Then $Y_{0}$ is isomorphic to the orbit space $V / G$ and $Z_{0}$ is isomorphic to $W / H$. Strub [Str82] has shown that if there is a diffeomorphism of neighborhoods of the origins in $Y_{0}$ and $Z_{0}$, then the $G$ and $H$ actions are quasi-isomorphic. Prill [Pri67] showed the analogue of Strub's result in the case of complex representations $U$ of finite complex groups $K$ not containing pseudoreflections. Here one considers the complex variety $U / K$ corresponding to $\mathbb{C}[U]^{K}$. Gottschling [Got69] and Prill [Pri67] proved that biholomorphisms of $U / K$ lift to equivariant biholomorphisms of $U$, again assuming that $K$ contains no pseudoreflections. In [Los01] and [KLM03] there are results about lifting of isomorphisms of complex quotients $U / G \simeq U^{\prime} / H$ where $U$ and $U^{\prime}$ are complex $G$ and $H$-modules, respectively. Lyashko [Lya83] showed that lifting holds in case $G=H$ is a Weyl group and $U=U^{\prime}$ is the complexification of the standard real representation. Losik [Los01] proves that diffeomorphisms of $Y_{0}$ lift to diffeomorphisms of $V$ which preserve $G$-orbits. We give new proofs of the lifting result of [KLM03] and of the main result of [Str82]

We thank K. Abe for posing questions leading to this note and for making us aware of [Str82].

\section{ISOMORPHISMS OF SPACES OF INVARIANTS}

Let $p_{1}, \ldots, p_{m}$ be homogeneous generators of $\mathbb{R}[V]^{G}$ and let $q_{1}, \ldots, q_{n}$ be homogeneous generators of $\mathbb{R}[W]^{H}$. Let $d_{j}$ denote the degree of $p_{j}, j=1, \ldots, m$ and let $e_{j}$ denote the degree of $q_{j}$, $j=1, \ldots, n$. Let $p=\left(p_{1}, \ldots, p_{m}\right): V \rightarrow \mathbb{R}^{m}$ and $q=\left(q_{1}, \ldots, q_{n}\right): W \rightarrow \mathbb{R}^{n}$. Let $Y_{0}$ denote $p(V)$ and let $Z_{0}$ denote $q(W)$. Then $Y_{0}$ and $Z_{0}$ are closed and semialgebraic where $Y_{0} \simeq V / G$ and $Z_{0} \simeq W / H$. We may identify $Y$ and $Z$ with the Zariski closures of $Y_{0}$ in $\mathbb{R}^{m}$ and of $Z_{0}$ in $\mathbb{R}^{n}$. Since the $p_{i}$ are homogeneous, $Y_{0}$ is stable under the $\mathbb{R}^{*}$ action on $\mathbb{R}^{m}$ which sends $t \in \mathbb{R}^{*}$ and $y=\left(y_{1}, \ldots, y_{m}\right) \in \mathbb{R}^{m}$ to $t \cdot y:=\left(t^{d_{1}} y_{1}, \ldots, t^{d_{m}} y_{m}\right)$. It follows that $Y$ is stable under this action. Similarly we have an action of $\mathbb{R}^{*}$ on $\mathbb{R}^{n}$ which preserves $Z_{0}$ and $Z$. Let $V_{\mathbb{C}}=V \otimes_{\mathbb{R}} \mathbb{C}$ denote the complexification of $V$. Then $p: V_{\mathbb{C}} \rightarrow \mathbb{C}^{m}$ has image the complex variety $Y_{\mathbb{C}}$ corresponding to $\mathbb{C}\left[V_{\mathbb{C}}\right]^{G}$. Moreover, $Y$ is $Y_{\mathbb{C}} \cap \mathbb{R}^{m}$. The strata of $Y$ and $Y_{0}$ are the collections of points whose preimages in $V_{\mathbb{C}}$ have conjugate isotropy groups. There are clearly finitely many strata. We say that a stratum is codimension one if it is of codimension one in $Y_{0}$ or $Y$, as the case may be. Equivalently, the isotropy groups corresponding to the stratum have order 2 and are generated by reflections. The principal points of $Y$ and $Y_{\mathbb{C}}$ are those points whose inverse images in $V_{\mathbb{C}}$ have trivial isotropy groups.

Example 2.1. Let $V=\mathbb{R}$ and $G=\{ \pm 1\}$ acting by multiplication. Then $p=x^{2}: \mathbb{R} \rightarrow \mathbb{R}$. We have that $Y_{0}=\mathbb{R}^{+}$and $Y=\mathbb{R}$. The codimension one stratum of $Y$ is the origin and the principal points are $Y \backslash\{0\}$.

Example 2.2. Let $V=\mathbb{R}^{2}$ and $G=\mathbb{Z} / k \mathbb{Z}$ acting via rotations, $k \geq 2$. Let $x$ and $y$ be the usual coordinate functions on $V$ and set $z=x+i y$. Then the $G$-invariant polynomials are generated by $z \bar{z}$ and the real and imaginary parts of $z^{k}$. Thus $p_{1}=x^{2}+y^{2}, p_{2}=x^{k}-\left(\begin{array}{l}k \\ 2\end{array}\right) x^{k-2} y^{2}+\ldots$ and $p_{3}=k x^{k-1} y-\left(\begin{array}{l}k \\ 3\end{array}\right) x^{k-3} y^{3}+\ldots$ generate $\mathbb{R}[V]^{G}$. They satisfy the relation $p_{2}^{2}+p_{3}^{2}=p_{1}^{k}$. Hence $Y=\left\{\left(y_{1}, y_{2}, y_{3}\right) \in \mathbb{R}^{3} \mid y_{1}^{k}=y_{2}^{2}+y_{3}^{2}\right\}$ and one can show that $Y_{0}=\left\{\left(y_{1}, y_{2}, y_{3}\right) \in Y \mid y_{1} \geq 0\right\}$. If $k$ is odd, then $Y=Y_{0}$, while $Y$ and $Y_{0}$ differ if $k$ is even. Here the strata are again $\{0\}$ and $Y \backslash\{0\}$, so there are no codimension one strata.

Assume that we have a germ of a diffeomorphism $f: Y \rightarrow Z$ sending 0 to 0 . This means that $f$ extends to a smooth germ of a mapping from $\mathbb{R}^{m}$ to $\mathbb{R}^{n}$ sending $Y$ to $Z$ and similarly for $f^{-1}$. We assume that $f$ sends the closures of the codimension one strata of $Y$ onto the closures of the codimension one strata of $Z$. 
Lemma 2.3. Let $f: Y \rightarrow Z$ be a local diffeomorphism as above. Then $f$ maps principal points of $Y$ to principal points of $Z$.

Proof. Everything we do should be understood to happen in neighborhoods of 0 in $Y$ and $Z$ where $f$ and $f^{-1}$ are defined. Let $C$ and $D$ be the closures of the codimension one strata in $Y$ and $Z$, respectively. It follows from the Shepherd-Todd-Chevalley theorem that $y \in Y$ is a smooth point if and only if the isotropy group of a preimage $y_{0} \in V_{\mathbb{C}}$ is trivial or is generated by reflections. In the latter case let $r$ be one of the reflections in $G_{y_{0}}$. Then $y$ lies in the closure of the codimension one stratum consisting of points whose isotropy group is generated by $r$. Thus a point in $Y \backslash C$ is smooth in $Y$ if and only if it is principal. Hence the principal stratum of $Y$ consists of the smooth points not in $C$, so that $f$ induces an isomorphism of the principal points of $Y$ and $Z$.

Let $y \in Y$ be close to zero so that $f$ is defined on $(0,1] \cdot y$. For $t \in(0,1]$, let $f_{t}$ denote $t^{-1} \cdot f(t \cdot y)$. We say that $f$ is quasilinear if $f_{t}=f$ for all $t \in(0,1]$. In this case, of course, $f$ is the restriction of a global diffeomorphism of $Y$ and $Z$ which is induced by a polynomial mapping.

Lemma 2.4. Let $f: Y \rightarrow Z$ be a local diffeomorphism as above. Then $f_{t}(y)$ converges uniformly to a limit $f_{0}(y)$ as $t \rightarrow 0$ for $y$ in a neighborhood of 0 in $Y$. The mapping $f_{0}$ is a quasilinear polynomial isomorphism of $Y$ and $Z$ which preserves the closures of the codimension one strata.

Proof. There are invariant inner products on $V$ and $W$ and we may assume that $p_{1}$ and $q_{1}$ are the corresponding quadratic forms. Let $Y^{\prime}$ and $Z^{\prime}$ denote the principal points of $Y$ and $Z$, respectively. Then $Y^{\prime}$ and $Z^{\prime}$ are $\mathbb{R}^{*}$-stable and have finitely many components. The image $Z_{1} \subset Z$ of the principal points of $W$ is connected and open in $Z$ and it is $\mathbb{R}^{*}$-stable. Since $q(W)=Z_{0}$ is closed in $Z$, any limit point of $Z_{1}$ which is not in $Z_{1}$ is not a principal point. Thus $Z_{1}$ is a connected component of $Z^{\prime}$. It follows that $f^{-1} Z_{1}$ lies in and contains a neighborhood of 0 of a connected component $Y_{1}$ of $Y^{\prime}$. Let $y \in Y_{1}$ such that $f$ is defined on $(0,1] \cdot y$. Set $c(t)=f(t \cdot y)$. Then $c(t)=\left(c_{1}(t), \ldots, c_{n}(t)\right)$ is a curve in $Z_{0}$ and from [Los01, Lemma 2.1] we see that $c_{i}(t)$ vanishes at least to order $e_{i}$ at $t=0$. Thus $f_{t}(y)$ converges to a limit $f_{0}(y)$. Now consider the Taylor polynomial $T f$ of $f$ up to the maximum degree $s$ of the $q_{i}$. Then the $i$ th component $T_{i}(f)$ is a sum of monomials $\sum c_{\alpha} y^{\alpha}$ where $c_{\alpha} \in \mathbb{R}$ and $\alpha=\left(\alpha_{1}, \ldots, \alpha_{m}\right) \in \mathbb{N}^{m}$. Let $|\alpha|$ denote $d_{1} \alpha_{1}+\cdots+d_{m} \alpha_{m}$. Then our calculation shows that the sum $\sum_{|\alpha|<e_{i}} c_{\alpha} y^{\alpha}$ vanishes on $Y_{1}$. Since $Y_{1}$ is Zariski dense in $Y$ the sum vanishes on $Y$. We may change $f$ without changing its restriction to $Y$ so that $T_{i}(f)$ has nonzero coefficients only for monomials $y^{\alpha}$ with $|\alpha| \geq e_{i}$. Then the Taylor polynomial of $f$ up to degree $s$ induces the mapping $f_{0}$ and the convergence of $f_{t}$ to $f_{0}$ is uniform for $y \in Y$ in a neighborhood of 0 . Since $f_{0}$ is a limit of maps preserving the closures of the codimension one strata, $f_{0}$ does also, and $f_{0}$ is clearly quasilinear. It is also invertible, with inverse $\left(f^{-1}\right)_{0}$.

Remark 2.5. It is clear that $f_{0}$ induces a complex isomorphism of $Y_{\mathbb{C}}$ and $Z_{\mathbb{C}}$, also denoted $f_{0}$, preserving the closures of the codimension one strata. This follows from the fact that the Zariski closures of the strata of $Y_{0}$ in $Y_{\mathbb{C}}$ are the strata of $Y_{\mathbb{C}}$, and this correspondence preserves codimension [Sch80, 5.8].

Remark 2.6. In Examples 2.1 and 2.2 (with $W=V$ and $H=G$ ) the mapping $f_{0}$ is linear. This is obvious in Example 2.1 since the generators are all quadratic. Example 2.2 requires more work.

Corollary 2.7. Let $f_{0}: Y_{\mathbb{C}} \rightarrow Z_{\mathbb{C}}$ be a quasilinear isomorphism which induces an isomorphism of the closures of the codimension one strata. Then there is a quasi-isomorphism $F: V_{\mathbb{C}} \rightarrow W_{\mathbb{C}}$ which induces $f_{0}$. 
Proof. The proof of Lemma 2.3 shows that $f_{0}: Y_{\mathbb{C}} \rightarrow Z_{\mathbb{C}}$ induces an isomorphism of the principal points. Since it also preserves the closures of the codimension one strata, it has a biholomorphic lift $F: V_{\mathbb{C}} \rightarrow W_{\mathbb{C}}$ such that $F$ and $F^{-1}$ preserve orbits [KLM03]. Let $F_{t}(v)=t^{-1} F(t v)$ for $t \in \mathbb{R}^{*}$ and $v \in V$. Then $F_{t}$ is a continuous family of biholomorphic lifts of $f_{0}$, so that we must have $F_{t}=F$ for all $t$ and hence $F=\lim _{t \rightarrow 0} F_{t}$ is linear. Thus $F$ is a quasi-isomorphism.

Let $h \in H$. Set $W_{h}:=W_{h+} \oplus i W_{h-}$ where $W_{h \pm}$ denotes the \pm 1 -eigenspace of $h$.

Lemma 2.8. Let $z^{\prime} \in Z$. Then there is an $h \in H$ such that $z^{\prime} \in p\left(W_{h}\right)$.

Proof. Let $z \in W_{\mathbb{C}}$ such that $q(z)=z^{\prime}$. Since the polynomials making up $q$ have real coefficients, $q(z)=\overline{q(z)}=q(\bar{z})$. Since $q(z)=q(\bar{z})$, there is an $h \in H$ such that $\bar{z}=h z$. Then $z=w_{1}+i w_{-1}$ for some $w_{1} \in W_{h+}$ and $w_{-1} \in W_{h-}$.

Theorem 2.9. Let $f: Y \rightarrow Z$ be a local diffeomorphism sending 0 to 0 which preserves the closures of the codimension one strata. Then there is a quasi-isomorphism $F: V \rightarrow W$.

Proof. We have shown that there is a quasi-isomorphism $F: V_{\mathbb{C}} \rightarrow W_{\mathbb{C}}$ which induces $f_{0}$ on $Y$. Thus $F(V)$ has to be an $H$-stable linear subspace of $W_{\mathbb{C}}$ which maps to $Z$. Since $H$ is finite, it follows from Lemma 2.8 that $F(V)=W_{h}$ for some $h \in H$ such that $h^{2}=1$ and such that $h$ is central in $H$ (else $F(V)$ is not $H$-stable). Since $W_{h}$ is $H$-equivariantly isomorphic to $W$, we see that $V$ and $W$ are quasi-isomorphic.

Remark 2.10. Let $F, h$ and $f$ be as in the proof above. The linear mapping from $\mathbb{R}[W]^{H}$ to $\mathbb{R}[W]^{H}$ which sends $f\left(w_{1}+w_{-1}\right)$ to $f\left(w_{1}+i w_{-1}\right)$ induces a graded automorphism of $\mathbb{R}[W]^{H}$, hence a "linear" automorphism $\varphi$ of $Z$. Then $\varphi$ identifies $Z_{0}$ and $q\left(W_{h}\right)$. Our diffeomorphism $f$ is just a local diffeomorphism of $Y$ preserving $Y_{0}$ followed by the identification of pairs $Y_{0} \subset Y$ with $q\left(W_{h}\right) \subset Z$ induced by the quasi-isomorphism $F$.

Our techniques establish the following theorem of Strub [Str82].

Theorem 2.11. Let $f: Y_{0} \rightarrow Z_{0}$ be a local diffeomorphism sending 0 to 0 . Then there is a quasi-isomorphism $F: V \rightarrow W$.

Proof. The principal points of $Y_{0}$ are exactly the points where it is locally a smooth manifold without boundary and the codimension one strata are exactly the boundary of the points where $Y_{0}$ is locally a manifold with boundary. Thus the analogues of Lemmas 2.3 and 2.4 hold for $f$ and $f_{0}$. Since $f_{0}$ must automatically give an isomorphism of $Y$ and $Z$, the proof of Theorem 2.9 goes through.

Finally, we prove a result on lifting global isomorphisms generalizing [Los01, Theorem 3.4].

Theorem 2.12. Let $f: Y_{0} \rightarrow Z_{0}$ be a diffeomorphism. Then there is a lift $F: V \rightarrow W$ of $f$ where $F$ maps $G$-orbits to $H$-orbits.

Proof. Let $f_{t}$ be as in Lemma 2.4. Then there is a quasi-isomorphism $F^{\prime}: V \rightarrow W$ covering $f_{0}$. Now $f_{0}^{-1} f_{t}$ is an isotopy of the identity of $Y_{0}$. By the isotopy lifting theorem for finite groups [Bie75] or [Sch80], we may lift $f_{0}^{-1} f_{t}$ to an equivariant isotopy $F_{t}: V \times[0,1] \rightarrow V$. Then $F^{\prime} \circ F_{1}: V \rightarrow W$ is the desired lift of $f$.

\section{Lifting Biholomorphisms}

We have used lifting results from [KLM03]. The proofs there use results about connections or braid groups. Here we sketch how one can use the slice theorem for finite group actions to obtain their lifting result.

We change notation. Let $G \subset \mathrm{GL}(V)$ and $H \subset \mathrm{GL}(W)$ be finite subgroups where $V$ and $W$ are finite dimensional complex vector spaces. Let $Y$ denote $V / G$ (the variety associated to 
$\mathbb{C}[V]^{G}$ ) and let $Z$ denote $W / H$. If $g \in G$ is a pseudoreflection, let $r_{g}$ denote its order and let $V^{<g>} \subset V$ denote the points of $V^{g}$ whose isotropy group is generated by $g$. Then $C$, the union of the strata of codimension one, is a disjoint union $C_{1} \cup \cdots \cup C_{k}$ where each $C_{j}$ is the image of a subset $V^{<g_{j}>}$ for a pseudoreflection $g_{j}, j=1, \ldots, k$. To the component $C_{j}$ we associate the number $r_{j}:=r_{g_{j}}$. Similarly we have the union $D$ of the codimension one strata of $Z$ and a decomposition $D=D_{1} \cup \cdots \cup D_{l}$ and pseudoreflections $h_{1}, \ldots, h_{l} \in H$ with orders $s_{1}, \ldots, s_{l}$ such that $D_{j}$ is the image of $W^{<h_{j}>}, j=1, \ldots, l$.

Theorem 3.1. Suppose that $k=l$ and that $f: Y \rightarrow Z$ is a biholomorphism which sends each $\overline{C_{i}}$ onto a $\overline{D_{j}}$ such that $r_{i}=s_{j}$. Then there is a biholomorphic map $F: V \rightarrow W$ such that $F$ and $F^{-1}$ send orbits to orbits, and $F$ induces $f$.

Remark 3.2. It is easy to see that the conditions of the theorem are necessary for $F$ to exist.

Proof of Theorem 3.1. We use a version of analytic continuation. Let $V_{1}$ denote the union of the reflection hyperplanes of $V$ and let $V_{2}$ denote the union of the fixed subspaces $V^{K}$ of subgroups $K$ of $G$ such that $\operatorname{codim}_{V} V^{K} \geq 2$. Let $Y_{j}$ denote the image of $V_{j}$ in $Y, j=1$, 2. Similarly we have subsets $W_{j} \subset W$ and $Z_{j} \subset Z, j=1,2$. Let $v \in V_{1} \backslash V_{2}$, let $g$ denote a reflection which generates the isotropy group of $v$ and let $w \in W^{h} \backslash W_{2}$ be a point lying above $f(p(v))$ where $h$ is a reflection generating the isotropy group of $w$. By hypothesis, $g$ and $h$ both have the same order, say $r$. By the slice theorem there is a ball $B_{1}$ about $v$ in $V^{g}$ and a ball $B_{2}$ in $\mathbb{C}$ around 0 such that a $G$-neighborhood of $v$ is isomorphic to $G \times{ }^{(g)}\left(B_{1} \times B_{2}\right)$ where $(g)$ denotes the subgroup generated by $g$. Here $g$ acts trivially on $B_{1}$ and acts on $B_{2}$ via multiplication by a primitive $r$ th root of unity. We use the notation $\left[g_{1}, x, y\right]$ to denote the point in $G \times{ }^{(g)}\left(B_{1} \times B_{2}\right)$ corresponding to $g_{1} \in G, x \in B_{1}$ and $y \in B_{2}$. Then the quotient mapping sends a point $\left[g_{1}, x, y\right]$ to $\left(x, y^{r}\right)$ for $x \in B_{1}$ and $y \in B_{2}$. Similarly, there are balls $B_{1}^{\prime}$ and $B_{2}^{\prime}$ about $w$ in $W^{h}$ and $0 \in \mathbb{C}$ such that the quotient mapping sends $\left.\left[h_{1}, x^{\prime}, y^{\prime}\right)\right] \in H \times{ }^{(h)}\left(B_{1}^{\prime} \times B_{2}^{\prime}\right)$ to $\left(x^{\prime},\left(y^{\prime}\right)^{r}\right), h_{1} \in H, x^{\prime} \in B_{1}^{\prime}, y^{\prime} \in B_{2}^{\prime}$. The hypotheses on $f$ show that for $(x, y) \in B_{1} \times B_{2}^{r}, f(x, y)=\left(f_{1}(x, y), f_{2}(x, y)\right) \in B_{1}^{\prime} \times\left(B_{2}^{\prime}\right)^{r}$ where $f_{2}$ vanishes when $y=0$ and the derivative of $f_{2}$ in $y$ has rank 1 along the zero set of $y$. It follows that, locally, $f_{2}(x, y)$ can be written as $m(x, y) y$ where $m(x, 0)$ does not vanish. Thus $f_{2}\left(x, y^{r}\right)$ has $r$ holomorphic $r$ th roots along $B_{1} \times\{0\}$, so that we have $r$ holomorphic lifts of $f$ in a neighborhood of $v$ which send $v$ to $w$. The lifts are distinguished by their values at any point $(x, y) \in B_{1} \times B_{2}$ where $y \neq 0$.

Now let $\gamma(t), 0 \leq t \leq 1$, be a continuous curve in $V \backslash V_{2}$ starting at a base point $v_{0}$. Let $F_{0}$ be a germ of a holomorphic map from $V \rightarrow W$ which covers $f$. Since $W \backslash W_{1} \rightarrow Z \backslash Z_{1}$ is a cover, we have an analytic continuation of $F_{0}$ to $F_{t}$ at $\gamma(t)$ as long as $\gamma(t)$ never leaves $V \backslash V_{1}$. However, our argument above shows that we can continue $F$ even if $\gamma(t)$ lands in $V_{1} \backslash V_{2}$. Thus we may construct a continuous family $F_{t}$ of lifts of $f$ along $\gamma(t)$. The family is uniquely determined by $F_{0}$. Now $V \backslash V_{2}$ is simply connected, so that $F_{1}$ only depends upon $\gamma(1)$. Thus we have a lift of $f$ to $V \backslash V_{2}$. Since $V_{2}$ has codimension 2 in $V$, our lift extends to all of $V$.

We have shown that there is a global lift $F$ of $f$, and similarly there is a global lift $F^{-1}$ of $f^{-1}$. Now for any $g \in G, F \circ g \circ F^{-1}$ is an automorphism of $W$ which covers the identity on $W / H$. Thus it must agree with an element of $H$. It follows that $g \mapsto F \circ g \circ F^{-1}$ gives an isomorphism of $G$ and $H$, and that $F$ and $F^{-1}$ send orbits to orbits.

\section{REFERENCES}

[Bie75] Edward Bierstone, Lifting isotopies from orbit spaces, Topology 14 (1975), no. 3, $245-252$.

[Got69] Erhard Gottschling, Invarianten endlicher Gruppen und biholomorphe Abbildungen, Invent. Math. 6 (1969), 315-326.

[KLM03] Andreas Kriegl, Mark Losik, and Peter W. Michor, Tensor fields and connections on holomorphic orbit spaces of finite groups, J. Lie Theory 13 (2003), no. 2, 519-534. 
[Los01] M. V. Losik, Lifts of diffeomorphisms of orbit spaces for representations of compact Lie groups, Geom. Dedicata 88 (2001), no. 1-3, 21-36.

[Lya83] O. V. Lyashko, Geometry of bifurcation diagrams, Current problems in mathematics, Vol. 22, Itogi Nauki i Tekhniki, Akad. Nauk SSSR Vsesoyuz. Inst. Nauchn. i Tekhn. Inform., Moscow, 1983, pp. 94129.

[Pri67] David Prill, Local classification of quotients of complex manifolds by discontinuous groups, Duke Math. J. 34 (1967), 375-386.

[Sch80] Gerald W. Schwarz, Lifting smooth homotopies of orbit spaces, Inst. Hautes Études Sci. Publ. Math. (1980), no. 51, 37-135.

[Str82] Rainer Strub, Local classification of quotients of smooth manifolds by discontinuous groups, Math. Z. 179 (1982), no. $1,43-57$.

Department of Mathematics, Brandeis University, Waltham, MA 02454-9110

E-mail address: schwarz@brandeis.edu 